\title{
Posterior reversible encephalopathy syndrome (PRES) in a woman who developed intrapartum eclampsia
}

\begin{abstract}
Posterior Reversible Encephalopathy Syndrome (PRES) is a clinicoradiological term which implies that the patient has a reversible vasogenic edema of the brain. Its clinical features include confusion, seizures, headache and visual disturbance. We present here a case of a woman who acquired posterior reversible encephalopathy syndrome after developing eclampsia intrapartum. She was treated with Magnesium sulphate to stop her seizures and intravenous labetalol to control blood pressure (B.P). The patient regained consciousness 3 days after the delivery of her baby. However, she complained of decreased visual acuity, which improved significantly over a period of three weeks. The Magnetic Resonance Imaging (MRI) of the brain showed T2 weighted image (T2W1) symmetrical cortical high signals in the bilateral parietooccipital cortex and subcortical white matter.
\end{abstract}

Keywords: posterior reversible encephalopathy syndrome (PRES), eclampsia, emergency obstetric care unit (EMOC)
Volume 5 Issue 4 - 2016

\author{
Syed Hussain Abbas,' Mohammad Naeem, \\ Muhammad ljaz,' Muhammad Zia UI Islam \\ khan $^{3}$ \\ 'Department of Medicine, Khyber Teaching Hospital, Pakistan \\ ${ }^{2}$ Department of Internal Medicine, Khyber Teaching Hospital, \\ Pakistan \\ ${ }^{3}$ Department of Community Medicine, Khyber Medical College, \\ Pakistan \\ ${ }^{4}$ Department of Internal Medicine, Robert Packer Hospital, USA
}

Correspondence: Syed Hussain Abbas, Department of
Medicine, Khyber Teaching Hospital, Peshawar, Pakistan, Tel
+923459133948, Email drsyedabbas88@gmail.com

Received: December 0I, 2016 | Published: December 30, 2016
Abbreviations: PRES, posterior reversible encephalopathy syndrome; BP, blood pressure; MRI, magnetic resonance imaging; $\mathrm{CT}$, computed tomography; T2W1, T2 weighted image; EMOC, emergency obstetric care unit, GCS, glasgow comma scale; DW-MRI, diffusion-weighted magnetic resonance imaging; MRA, magnetic resonance angiography; MRV, magnetic resonance venography; APGAR, appearance pulse grimace activity respiration

\section{Introduction}

Posterior reversible encephalopathy syndrome is a condition with a spectrum of different symptoms including altered mental status, vision loss, headache and seizures. The etiology is still unclear but it has been associated with immuno suppressants, eclampsia, systemic lupus erythematosis, infection, sepsis and shock. We report here a case of PRES which has presented with its typical clinical, imaging and outcome findings; endorsing eclampsia as one of the causes of PRES. ${ }^{1,2}$

\section{Case presentation}

A 25year old woman presented with tonic clonic seizures to our emergency department in a tertiary care facility. She developed these seizures during labor in a basic Emergency Obstetric Care unit (EmOC). In the emergency department, we assessed and treated her according to the Airway, Breathing, Circulation, Disability, Exposure (ABCDE) approach. ${ }^{3}$ An intravenous access was secured, and she was given Magnesium sulphate to stop the seizure activity. On clinical examination her blood pressure (B.P) was 170/110mm of $\mathrm{Hg}$ and urinalysis showed proteinuria of greater than 3grams suggesting eclampsia. Her blood pressure was controlled and maintained in the range of $140-160 / 90-110 \mathrm{~mm}$ of $\mathrm{Hg}$ by administering intravenous (IV) labetalol. After the patient was stabilised, the baby was delivered at full term vaginally and his APGAR (Appearance, Pulse, Grimace,
Activity, Respiration) score was found to be in the normal range i.e. 7/10; both at birth and after five minutes. The patient was irritable with a Glasgow comma scale (GCS) of 10. Therefore, she was shifted to the intensive care unit to provide further care. After two days, she developed a high grade fever of $400 \mathrm{C}$. To find out the cause, a transabdominal and transvaginal ultrasound was performed which showed retained products of conception in the uterus; these were removed with evacuation and curettage. A combination of antibiotics (ceftriaxone and metronidazole) was also started to treat the infection. The fever subsequently subsided after 4days. The patient gained consciousness after three days and complained of impaired vision. Her visual acuity was found to be $6 / 60$ in both eyes. The visual field examination showed inferior left homonymous quadrantanopia (Figure 1) and ophthalmoscopy showed normal fundi. Her blood pressure was assessed to be $158 / 95 \mathrm{~mm}$ of $\mathrm{Hg}$, which is a Grade 1 hypertension ${ }^{4}$ and therefore, the patient was started on amlodipine.

A Computed Tomography (CT) scan of the brain without contrast was done which showed low attenuation areas in bilateral parieto occipital regions with effaced subarachnoid spaces. Magnetic Resonance Imaging (MRI) of the brain depicted T2 weighted image (T2W1) symmetrical cortical high signals in the bilateral parieto occipital cortex and subcortical white matter (Figures 2) (Figure 3), suggesting Posterior Reversible Encephalopathy Syndrome (PRES). Magnetic Resonance Angiography (MRA) and Magnetic Resonance Venography (MRV) turned out to be normal.

Posterior reversible encephalopathy syndrome is associated with many clinical and imaging differential diagnoses. ${ }^{5}$ The clinical differentials include trauma, infective encephalitis, ictal/post-ictal state, neoplasm, and cerebrovascular accident, while the imaging differentials are Progressive Multifocal Leukoencephalopathy (PML), severe hypoglycaemia, posterior circulation stroke, gliomatosis cerebri and sagittal sinus thrombosis. The laboratory results were 
correlated with history, physical examination and specific findings on imaging of the brain, which ruled out all the other differentials and suggested the diagnosis of posterior reversible encephalopathy syndrome.

The patient showed significant improvement in her visual acuity over a period of three weeks. The clinical examination was unremarkable except for her raised blood pressure which was in the range of Grade 1 hypertension. The patient was advised to take amlodipine and counselled regarding diet and exercise for the long term control of her blood pressure. ${ }^{6}$

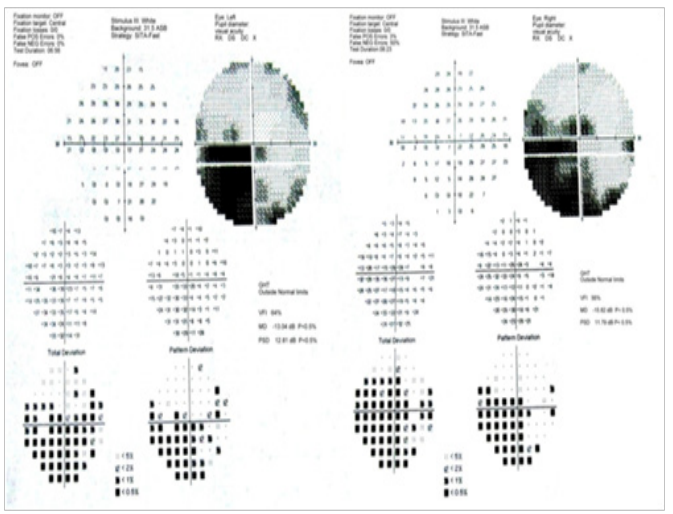

Figure I Perimetry depicting left inferior quadrantonopia.

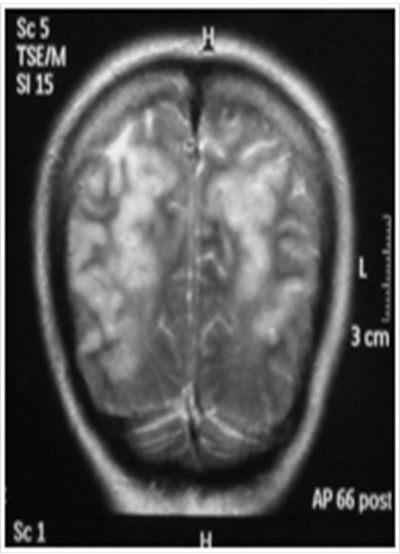

Figure 2 (Coronal view): MRI of brain Showing T2WI symmetrical cortical high signals in bilateral parieto-occipital cortex and subcortical white matter.

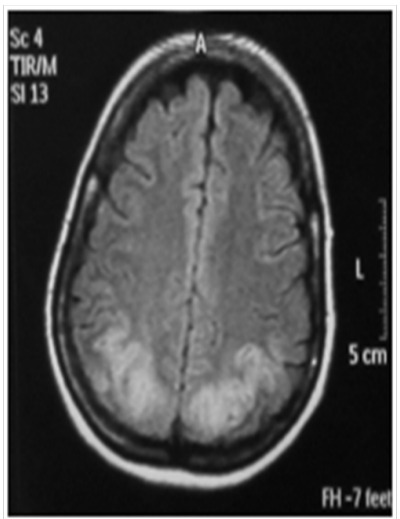

Figure 3 (Axial view): MRI of brain Showing T2WI and FLAIR symmetrical cortical high signals in bilateral parieto-occipital cortex and subcortical white matter.

\section{Discussion}

Posterior reversible encephalopathy syndrome has been reported frequently all over the world. ${ }^{7-9}$ It is a neurological condition which is diagnosed on the basis of clinical and radiological signs. The clinical features include confusion, seizures, headache and visual disturbances. Investigations such as magnetic resonance imaging usually reveal hyper intense signals at cortical and subcortical areas of posterior distribution (Figures 1) (Figure 2) but other areas such as frontal lobe, brainstem, basal ganglia and cerebellum may also be involved. ${ }^{10,11}$ There are three notable patterns of posterior reversible encephalopathy syndrome i.e. holohemispheric, superior frontal sulcus and primary parietal-occipital; all of these follow the watershed areas of distribution between major cerebral arteries. ${ }^{12}$ Although Diffusion-Weighted Magnetic Resonance Imaging (DW-MRI) is of great value in detecting infarcts early on and differentiating vasogenic from cytotoxic edema, it was not available in our hospital..$^{13}$

In the literature, posterior reversible encephalopathy syndrome is associated with renal failure, immunosuppression, chemotherapeutic agents, eclampsia, SLE and HIV..$^{14}$ In this case, the patient developed posterior reversible encephalopathy syndrome after eclampsia intrapartum.

The pathophysiology regarding PRES is explained by vasogenic and cytotoxic theory. ${ }^{15}$ The vasogenic theory states that due to loss of cerebral autoregulation, cerebral vessels fail to constrict in response to high mean arterial blood pressure and thus result in vasodilation, causing diffusion of plasma proteins outside the vasculature which results in cerebral edema; the loss of autoregulation due to high blood pressure may also disrupt the blood brain barrier and increase its permeability.

On the other hand, cytotoxic theory suggests that an abrupt and sharp increase in blood pressure results in severe vasoconstriction and hypoxic injury to the endothelial cells resulting in cytotoxic edema. ${ }^{16}$

\section{Acknowledgements}

None.

\section{Conflict of interest}

The author declares no conflict of interest.

\section{References}

1. Papoutsis D, El-Attabi N, Sizer A. Postpartum posterior reversible encephalopathy syndrome (PRES) in a twin pregnancy complicated by preeclampsia-eclampsia: case report. Clin exp Obstet \& Gynecol. 2014;41(3):351-353.

2. Wahab KW, Sanya EO, Ademiluyi BA, et al. Posterior reversible encephalopathy syndrome complicating postpartum eclampsia in a Nigerian:Case report. Niger Postgrad Med J. 2014;21(3):266-268.

3. Thim T, Krarup NHV, Grove EL, et al. Initial assessment and treatment with the Airway, Breathing, Circulation, Disability, Exposure (ABCDE) approach. Int J Gen Med. 2012;5:117-121

4. Whitworth JA. World Health Organization (WHO)/International Society of Hypertension (ISH) statement on management of hypertension. $J$ Hypertens. 2003;21(11):1983-1992.

5. Hobson EV, Craven I, Blank SC. Posterior reversible encephalopathy syndrome: A truly treatable neurologic illness. Perit Dial Int. 2012;32(6):590-594. 
6. Everett CM, Turner B, Lobo M. Posterior reversible encephalopathy syndrome in (low renin) essential hypertension. $J R$ Soc Med. 2007;100(11):522-523.

7. Zahir MN. Posterior reversible encephalopathy syndrome: case report. Reactions. 2013;1436:26.

8. Jaiswal A. Posterior reversible encephalopathy syndrome: case report. Reactions. 2015;1543:194-21.

9. Nixon NA. Posterior reversible encephalopathy syndrome: case report. Reactions. 2015;1533:93-10.

10. Ergun T, Lakadamyali H, Yilmaz A. Recurrent posterior reversible encephalopathy syndrome in hypertensive patient with end stage renal disease. Diagn Interv Radiol. 2008;14(4):182-185.

11. Postma IR, Slager S, Kremer HP, et al. Long-term consequences of the posterior reversible encephalopathy syndrome in eclampsia and preeclampsia:a review of the obstetric and nonobstetric literature. Obstet Gynecol Surv. 2014;69(5):287-300.
12. Bartynski WS, Boardman JF. Distinct imaging patterns and lesion distribution in posterior reversible encephalopathy syndrome. AJNR Am J Neuroradiol. 2014;28(7):1320-1327.

13. Demirel İ, KavakBS, Özer AB, et al. An intensive care approach to posterior reversible encephalopathy syndrome (PRES):An analysis of 7 cases. J Turk Ger Gynecol Assoc. 2014;15(4):217-221.

14. Neki NS, Mani T. Posterior reversible encephalopathy syndrome presenting with seizures. Natl Med J India. 2013;26(5):282-283.

15. Sasson SC, Oon A, Chagantri J, et al. Posterior reversible encephalopathy syndrome (PRES) in an HIV-1 infected patient with disseminated varicella zoster virus:a case report. BMC infectious diseases. 2013;13(1):1-5.

16. Stott VL, Hurrell MA, Anderson TJ. Reversible posterior leukoencephalopathy syndrome, a misnomer reviewed. Internal med J. 2005;35(2):83-90. 